\title{
Research on MPPT Algorithm Based on Mixed Strategy
}

\author{
Yu Chai $^{1}$, Xu Fang ${ }^{2}$ \\ ${ }^{1,2}$ Xi'an University of Science and Technology, Xi'an 710054, China
}

\begin{abstract}
Based on the mathematical model of PV array and its output characteristics, a hybrid control strategy combining fixed step method and variable coefficient approximation method is proposed. The method is followed by a fixed step in the search of the maximum power point. When the slope of the PU curve changes for the first time, the search interval is fixed in an initial step, and then the search is gradually approximated to maximum power point in the section. The method is analyzed theoretically and modeled in Matlab / Simulink environment, and compared with the variable step perturbation observation method. The simulation results show that the proposed method can quickly and accurately track the maximum power point under the standard condition and the sudden change of light intensity, and has faster tracking speed.
\end{abstract}

Keywords: PV array; hybrid strategy; fixed step; progressive approximation method; maximum power point

\section{Introduction}

Traditional fossil energy consumption brought about environmental pollution, greenhouse effect and many other environmental problems, it has been a great threat to human survival and development. Solar energy is a clean, safe, reliable and well-stocked renewable energy source that has become a major alternative to traditional fossil fuels. The output voltage and output current of the PV array vary with the temperature, light intensity and other external environment changes, and at a particular voltage in order to make the output power value to the maximum. Therefore, it is necessary to add the maximum power tracking module to the PV array to stabilize its output power at maximum. The variable perturbation observation method is a kind of control algorithm that easy to implement and widely used, but the algorithm itself has some defects, including the optimization speed is slow, also easy to oscillate at the maximum power point.

In this paper, based on the analysis of variable step perturbation observation method, a hybrid control strategy combining fixed step and variable coefficient approximation is proposed. The method can be divided into two steps, first to a fixed step to search. According to the P-U characteristic curve of the PV array, it is known that when the slope is changed for the first time during the search, the search range can be fixed within an initial step range. In this range, the gradually approximation of the variable coefficients is performed. Compared with the perturbation observation method with slope as variable step size, the method has its advantages both in tracking speed and eliminating oscillation.

\section{The output curve of photovoltaic cells}

Under standard test conditions(1.illumination $1000 \mathrm{~W} / \mathrm{m}^{2} ; 2$.temperature $25^{\circ} \mathrm{C} ; 3$.spectral characteristics AM1.5 standard spectrum), according to $I_{s c}, U_{o c}$, $I_{m}, U_{m}$ parameters can get the output curve of the current.

$$
\begin{gathered}
I_{L}=I_{s c}\left[1-C_{1}\left(e^{\frac{U_{m}}{C_{2} U_{o c}}}-1\right)\right] \\
C_{1}=\left(1-\frac{I_{m}}{I_{s c}}\right) e^{\frac{-U_{m}}{C_{2} U_{o c}}} \\
C_{2}=\left(\frac{U_{m}}{U_{o c}}-1\right)\left[\ln \left(1-\frac{I_{m}}{I_{s c}}\right)\right]^{-1}
\end{gathered}
$$

The current output curve in the above formula is the standard test condition, and when the temperature and light intensity change does not equal to the reference temperature and the reference light intensity, this formula is not applicable, it is need to amend to describe the new characteristic curve. First, the temperature and light intensity are corrected.

$$
\Delta T=T-T_{r e f}, \quad \Delta S=\frac{S}{S_{r e f}}-1
$$

\footnotetext{
* Corresponding author: fangxu152@sina.com
} 


\section{$T_{r e f}$ (Reference temperature) \\ $S_{r e f}$ (reference light intensity)}

The parameters are optimized under temperature and light intensity correction.

$$
\begin{gathered}
I_{s c}^{\prime}=I_{s c} \frac{S}{S_{r e f}}(1+\alpha \Delta T) \\
U_{o c}^{\prime}=U_{o c}(1-\gamma \Delta T) \ln (1+\beta \Delta S) \\
I_{m}^{\prime}=I_{m} \frac{S}{S_{r e f}}(1+\alpha \Delta T) \\
U_{m}^{\prime}=U_{m}(1-\gamma \Delta T) \ln (1+\beta \Delta S)
\end{gathered}
$$

Typical value: $\alpha=0.0025 /{ }^{\circ} \mathrm{C}, \quad \beta=0.5$,

$$
\gamma=0.00288 /{ }^{\circ} \mathrm{C} \text {. }
$$

The optimized parameters are brought into the equation (1) can get the curve with different temperature and light conditions.

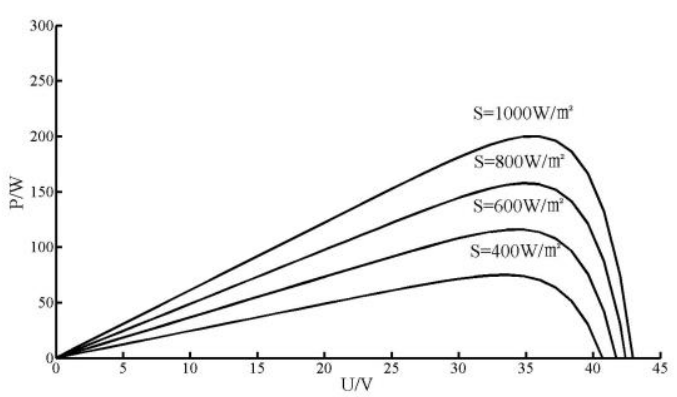

Fig.1. P-U characteristic curves of PV array for different insolations $\left(25^{\circ} \mathrm{C}\right)$

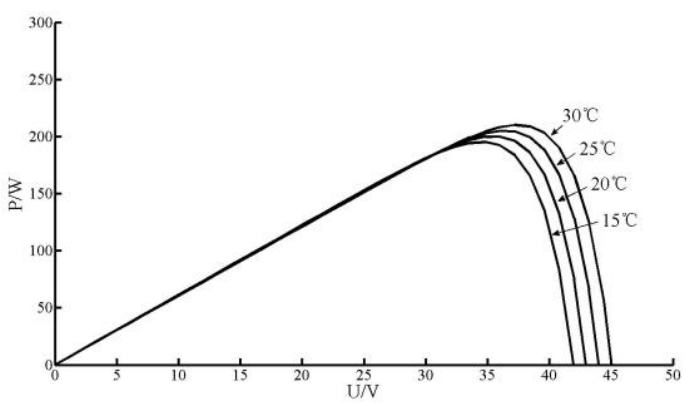

Fig.2. P-U characteristic curves of PV array for different temperature $\left(1000 \mathrm{~W} / \mathrm{m}^{2}\right)$

Figure 1 and Figure 2 shows that when the light intensity and temperature conditions change, the output curve will be offset. In a particular light intensity or temperature conditions under the curve, the voltage increases when the output power first monotonically increasing and then monotonically decreasing, at a certain voltage output power to reach the maximum. The search process of the maximum power point is essentially a singular optimization process.

\section{Research on Maximum Power Point Algorithm}

\section{1 variable step perturbation observation method}

Perturbation observation method is a control method that widely used in practice, the method by applying a disturbance to the system to determine the next step. If the power increases, then continue with the direction of disturbance, if the power is reduced, then change the direction of disturbance, until the step or power difference reaches the set threshold, then found that the maximum power point has been reached at this time. The traditional disturbance observation method takes a fixed step tracking. If the step size is too small, the tracking speed is slow; if the step size is too long, it will oscillate near the maximum power point, resulting in energy loss so that can not track the maximum power point.

The variable step perturbation observation method is optimized for the slope, with the slope as the variable step size coefficient. When away from the maximum power point, the slope $|k|=d P / d U>0$. At maximum power point, $|k|=d P / d U=0$,step size $D_{k}=k D_{k-1}$. Through the slope to optimize the step size, real-time change step size. When away from the maximum power point, the long size step is used to track quickly. When close to the maximum power point, the small size step is used to track stably. In order to quickly and accurately track, the process of finding the maximum power point need to constantly change the step size. Compared with the fixed-step perturbation method, the control effect of the variable-step perturbation observation method is more rapid and accurate.

\subsection{Mixed control strategy}

Based on the variable perturbation observation method, this paper proposes a hybrid control method combining fixed step method and variable coefficient continuous approximation method.

As shown in Fig. 3, a fixed step is selected to start the search with the PV array's P-U characteristic curve as a reference. The search process is performed from the left side of the curve to the right, and the slope of the curve is positive at the beginning of the search, and the slope of the curve is negative at the right of the maximum power point. 


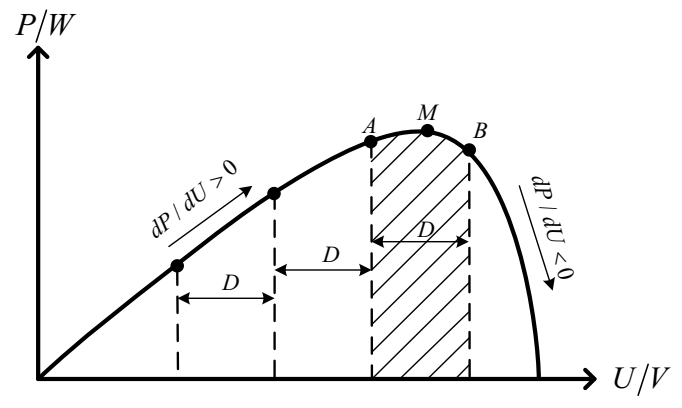

Fig.3. The first curve of search

In the stepwise search process, when the slope changes, that is, the first time from positive to negative (Figure 3, point $\mathrm{A}$ to point $\mathrm{B}$ ), indicating that at this time has crossed the maximum power point, the maximum power point should be in a fixed initial step within the area. Then change the search strategy, reverse to $\gamma D$ step to search, $\gamma$ range is from 0.5 to 1 ( $\gamma$ is 0.618 in this paper), so that within a certain range does not appear multiple searches.

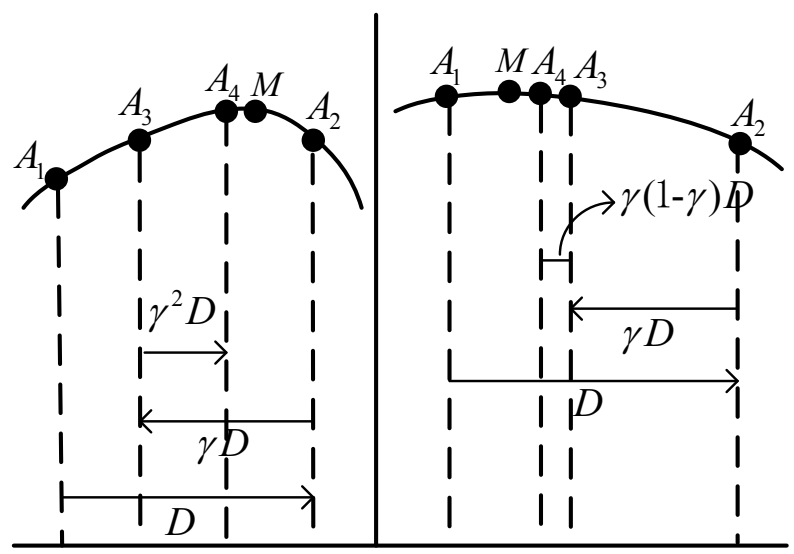

Fig.4. The second curve of search

If the slope does not change, indicating that the maximum power point has not been exceeded, continue with the direction of search, the maximum power point should be in the remaining $(1-\gamma) D$ range, select the step $\gamma(1-\gamma) D$ for the same direction search. If the slope changes, indicating that the maximum power point has been crossed, the maximum power point should be in the $\gamma D$ range, then the search direction should be changed to search in step $\gamma^{2} D$. The search interval is changed by the coefficient $\gamma$ and $1-\gamma$, and each search will divide the interval. The maximum power point in the large side of the interval, select the larger coefficient $\gamma$ to optimize the next search; in the small side of the interval, then select a smaller coefficient $1-\gamma$ to optimize the next search, making the search interval more rapid convergence. If the slope changes, that is, over the maximum power point, the step multiply $\gamma$, the opposite direction search. If the slope has not change ,the step multiply $1-\gamma$, the same direction to continue search, the algorithm flow chart shown in Figure 5.

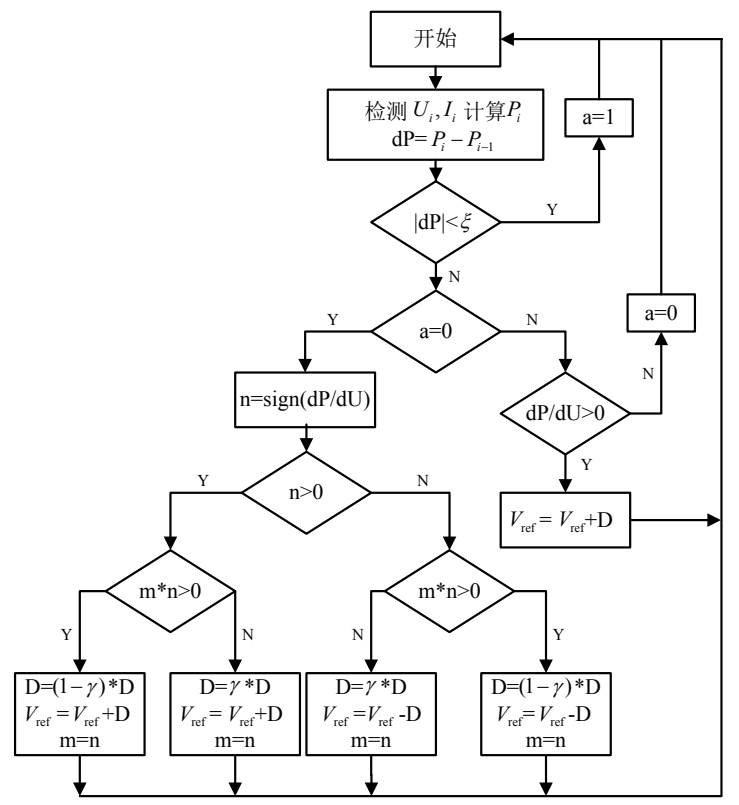

Fig.5. Flow chart of the algorithm

In the flow chart, $a$ is a numeric variable, the variable value can only be $\mathbf{0}$ or $\mathbf{1}$, the initial value is $\mathbf{1}$. When the slope is changed for the first time, that is, when the search process crosses the maximum power point, assign $a$ to $\mathbf{0}$, and the second step strategy is the variable coefficient approximation method. Until the power difference is less than the set threshold, then it is determined that the maximum power point has been reached, let $a$ be $\mathbf{1}$, and proceed to the next sampling. $m$, $n$ in the flow chart is a symbol variable, used to determine the last and the sampling slope of the positive and negative. $m$ or $n$ is greater than the value $\mathbf{0}$ indicates that the search is on the left side of the maximum power point, less than $\mathbf{0}$ indicates that the search for the maximum power point on the right.

\section{Simulink Modeling of Maximum Power Tracking System}

In order to verify the validity of the fixed step method combined with the variable coefficient approximation method proposed in this paper, building a model based on Matlab / Simulink. As shown in Figure 6, the model includes a photovoltaic cell module, an MPPT algorithm module, a typical boost circuit model, and a PWM module. Photovoltaic battery parameters selected a domestic production of the battery panel parameters ( $U_{o c}=200 \mathrm{~V}, I_{s c}=6.1 \mathrm{~A}, U_{m}=36 \mathrm{~V}, I_{m}=5.55 \mathrm{~A}, \mathbf{T}=25^{\circ} \mathrm{C}$ ). The MPPT module is programmed using the $\mathrm{S}$ function. 


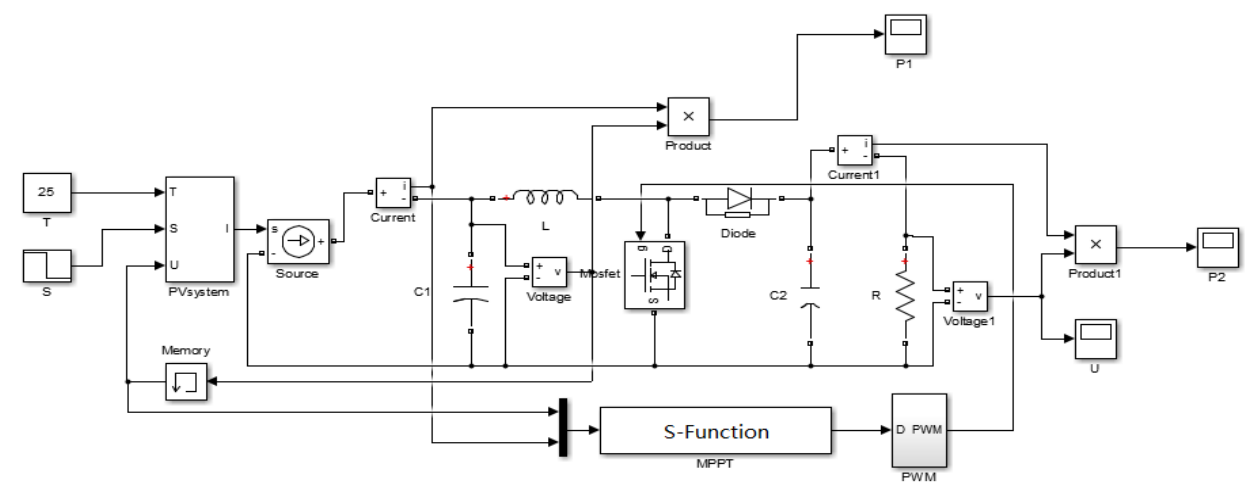

Fig.6. MPPT Simulation model of PV system

\section{Simulation results analysis}

The parameters of each component $\left(C_{1}=400 \mu \mathrm{F}\right.$, $\left.C_{2}=100 \mu \mathrm{F}, L=5 \mathrm{mH}, R=18 \Omega, P_{m}=200 \mathrm{~W}\right)$. The simulation time is set to $0.2 \mathrm{~s}$. The simulation results shown in Figure 7,8,9. Figure 7 is the voltage time curve under standard conditions, Figure 8 for the standard conditions of the power time curve, Figure 9 for the sudden change in the power time curve.

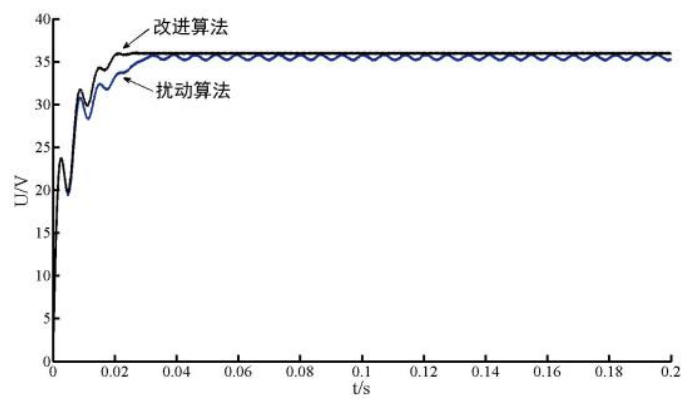

Fig.7. U-t curve under standard conditions $\left(1000 \mathrm{~W} / \mathrm{m}^{2}, 25^{\circ} \mathrm{C}\right)$

Figure 7 shows the output voltage and time of the PV panel. The theoretical maximum power point voltage of the panel is $36 \mathrm{~V}$. Under the standard conditions of light intensity of $1000 \mathrm{~W} / \mathrm{m}^{2}$ and temperature of $25^{\circ} \mathrm{C}$. The perturbation algorithm enters the maximum power point voltage oscillation interval around $0.03 \mathrm{~s}$, it can not accurately track the maximum power point voltage. The improved algorithm accurately tracks the maximum power point voltage at $0.02 \mathrm{~s}$, and there is no obvious oscillation. Compared with the traditional disturbance observation method, the improved algorithm has obvious advantages in eliminating oscillation.

Figure 8 shows the comparison between the improved algorithm and the variable step perturbation method. Under the standard conditions of light intensity $1000 \mathrm{~W} / \mathrm{m}^{2}$ and temperature $25{ }^{\circ} \mathrm{C}$, the two algorithms can quickly search for the maximum power point and have good stability, And the improved algorithm has faster tracking speed than the variable step perturbation observation method. The variable-length perturbation method is stable at $0.03 \mathrm{~s}$, while the hybrid algorithm in this paper traces the maximum power point at $0.02 \mathrm{~s}$, and the velocity is greatly improved.

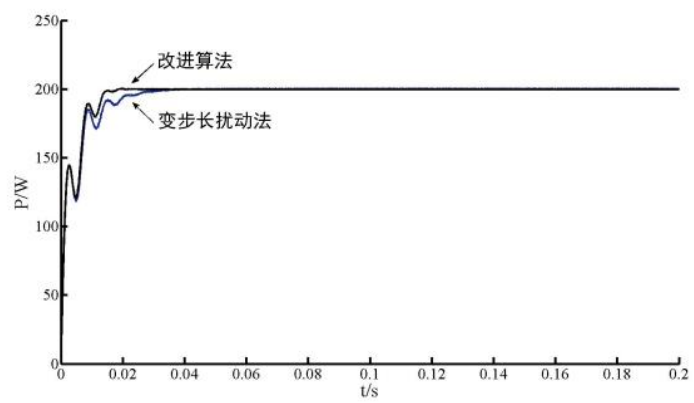

Fig.8. MPPT curve under standard conditions $\left(1000 \mathrm{~W} / \mathrm{m}^{2}, 25^{\circ} \mathrm{C}\right)$

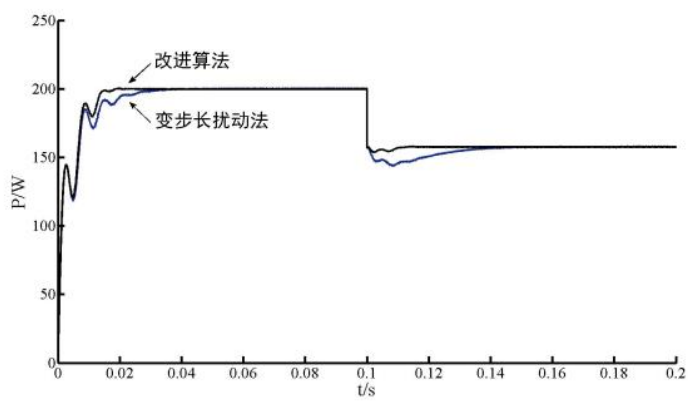

Fig.9. MPPT curve with the change of light intensity

In Fig. 9, the light intensity changes from 1000W/ $\mathrm{m}^{2}$ to $800 \mathrm{~W} / \mathrm{m}^{2}$ at $0.1 \mathrm{~s}$ when the light intensity is abruptly changed. The variable step perturbation method tracks the maximum power point again at $0.14 \mathrm{~s}$, and the improved algorithm tracts the maximum power point again at $0.11 \mathrm{~s}$. The improved algorithm still has a faster tracking rate in the event of a sudden change in light intensity.

\footnotetext{
*orresponding author: fangxu152@sina.com
} 


\section{Conclusion}

In order to track the maximum output power point of the photovoltaic cell accurately and quickly, on the basis of the variable step perturbation observation method, this paper proposes a hybrid control method combining the fixed step method and the variable coefficient approximation method.

The simulation results of Matlab / Simulink are used to compare the simulation results of different algorithms. The simulation results show that the fixed step method combined with the variable coefficient approximation method has its advantages both in the stability condition and in the light mutation condition, both in tracking speed and eliminating the oscillation.

\section{References}

1. Xiong Yuansheng, $\mathrm{Yu} \mathrm{Li}, \mathrm{Xu}$ Jianming, MPPT con trol of photovoltaic generation system combini-ng c onstant voltage method with $\mathrm{P} \& \mathrm{O}$ method [J]. Elect ric Power Automation Equipment,29(6):85-88,(200 9).

2. ZHU Tuofei,CHEN Guoding,Survey of MPPT for photovoltaic power generation[J].Chinese Journal of Power Sources,35(10):1322-1324,(2011).

3. LI Xingshuo, WEN Huiqing, Research on an improved $\beta$-based variable step MPPT algorithm $[\mathrm{J}]$. Power System Protection and Control,44(17):58-63, (2016).

4. XIE Yiwen,ZHU Jinrong,CONG Fangzhou,SHEN Deming,The Control of MPPT on the Photovoltaic System Based on the Improved Variable Step Perturbation Algorithm [J]. Power Electronics, 51(1):5 $3-55,(2017)$.

5. PAN Chao, MENG Tao, CAI Guowei,YIN Hang, Multiobjective optimization planning of generalize power in active distribution network[J]. Advanced Technology of Electrical Engineering and Energy , 35(3):69-75,(2016).

6. Huang Zhipeng, Pan Sanbo,MPPT control of model predictive control combined with golden section method [J]. Renewable Energy Resources, 34(12): 1766-1771,(2016).

7. LIU Fangrui, DUAN Shanxu, LIU Fei . Avariable step size INC MPPT method for PV systems [J] . IEEE Transactions on Industrial Electronics ,55(7): 2622-2628,(2008).

8. Piegari L, Rizzo R. Adaptive perturb and observe algorithm for photovoltaic maximum power point tracking[J].IET Renewable Power Generation,4(4): 317-328,(2010).

9. K.L.Lian, J.H.Jhang, I.S.Tian, A maximum power point tracking method based on perturband observe combined with particle swarm optimization , IEEE, vol4,pp. 626-633,(2014).

10. Chen Kun. Photovoltaic power generation systems MPPT control algorithm research [D.Chongqing, Chongqing University,(2013).

11.Zhu Minglian, Li Chensong, Chen Xin. A variable step size P\&O MPPT method for PV systems [J]. Power Electronics,44(1):20-22,(2010).

12. Wang Shuzheng, Li Xianyun, Application of a novel adaptive perturbation and observation method in MPPT strategy for photovoltatic power system [J]. Acta Energiae Solaris Sinica,37(9):2393-2396, (2016). 